Turk. J. Math. Comput. Sci.

12(2)(2020) 151-156

(C) MatDer

DOI : $10.47000 /$ tjmcs.793631

\title{
Behaviors of Eigenvalues and Eigenfunctions of The Singular Shrödinger Operator
}

\author{
RAuf Amirov $^{1}$ (D), SeVIM DuraK ${ }^{2, *}$ \\ ${ }^{1}$ Department of Mathematics, Faculty of Science, Sivas Cumhuriyet University, 58140, Turkey. \\ ${ }^{2}$ Department of Mathematics, Faculty of Science, Sivas Cumhuriyet University, 58140, Turkey.
}

Received: 11-09-2020 • Accepted: 26-11-2020

\begin{abstract}
Aвstract. Let us show the boundary value problem $L(q)$ with the $-y^{\prime \prime}+q(x) y=\lambda y$ differential equation in the $[0,1]$ interval, and the $y(0)=0, y(1)=0$ boundary conditions in $\sigma(x) \equiv \int_{0}^{x} q(t) d t$. It is important to examine this operator as the solution to many problems of quantum physics is closely linked to the learning of the spectral properties of the operator $L(q)$. Singular Shrödinger operators are characterized by the assumption that, in classical theory, the function $q(x)$ is not summable in the interval $[a, b]$ for example it has singularity that cannot be integrated in at least one of the end points of the interval or at one of its internal points, or that the interval $(a, b)$ is infinite interval.

In the present study, firstly, the operator of $L(q)$ will be proved to be well-defined in the class of distribution functions with first-order singularity, which is the larger class of functions. In the following step, the concepts of eigenvalue and eigenfunctions are defined for the well-defined $L(q)$ operator and the representations for their behaviour are obtained.
\end{abstract}

2010 AMS Classification: 34A55, 34B24, 34L05

Keywords: Singular Shrödinger operator, Eigenvalue, Eigenfunction.

\section{INTRODUCTION}

In the classical Sturm-Liouville operators theory produced by the differential expression $\ell(y)=-y^{\prime \prime}+q(x) y$ on $(a, b) \in \mathbb{R}$ it is generally assumed that the condition $q(x) \in L_{1, \ell o c}(a, b)$, that is, on $(a, b)$ of the $q(x)$ function, is summable in each compact subrange. Singular Sturm-Liouville operators are characterized by the assumption that $q(x)$ is not summable on $[a, b]$ or that on $(a, b)$ is infinite.

Spectral theory of differential operators has an important place in applied sciences and is widely used in various fields of mathematics, physics and mechanics. Particularly in quantum theory, the singular Shrödinger operator has many applications. For example, the energy levels of structures having hydrogen atoms and similar atoms are reduced to the problem of finding wave functions corresponding to these levels to learn the eigenvalues of the singular Shrödinger operator with the potential of Coulomb and the like and the behavior of the eigenfunctions corresponding to these eigenvalues. Therefore, in this study, we will learn the properties of singular Shrödinger operator with special type potential.

*Corresponding Author

Email addresses: emirov@cumhuriyet.edu.tr (R. Amirov),sdurak@cumhuriyet.edu.tr (S. Durak) 
Let us show with $\sigma^{\prime}(x)=q(x), \sigma(x) \in B V[0,1]$ the operator $L(q)$ produced by the

$$
\ell(y)=-y^{\prime \prime}+q(x) y=\lambda y, \quad \lambda=k^{2}
$$

differential equation and the $y(0)=0, y(1)=0$ boundary conditions on $[0,1]$. As it is known, $q(x)$ function has real value on $[0,1]$ and the spectra of this operatör is learned it can be integrated in Lebesque sense, and inverse problems are investigated according to different data.

Similar problems have been addressed in [1] and [7]. The $q \in W_{2}^{-1}(0,1)$-type potential of the differential expression produced by the differential expression of the operators $\ell(y)=-y^{\prime \prime}+q(x) y,|x|^{-\alpha}=q(x),\left(0<\alpha<\frac{3}{2}\right)$ case in the [7] study, in the case of $\alpha \in(1,2)$ is obtained in the [1] study. In addition [1,2] and [3-5] provide important information about the location of boundary value problems for singular differential equations. In particular in the work [1], $(0<$ $\alpha<2$ ), the problem of regularization was investigated for singular differential equations of type (1.1) and the problem of how to give boundary value conditions was examined.

\section{Property Of The Spectrum}

Let $L=L(u(x), h, H)$ boundary value problem produced by

$$
\ell y:=-y^{\prime \prime}+u^{\prime}(x) y=\lambda y, \quad 0<x<\pi, \lambda=k^{2}
$$

differential equation and

$$
U(y):=(\Gamma y)(0)-h y(0)=0, V(y):=(\Gamma y)(\pi)+H y(\pi)=0
$$

boundary conditions. Here, $h, H$ real numbers $u(x) \in B V[0, \pi]$ real value and continuous function in the points $x=$ $0, x=\pi, \lambda$ spectral parameter, $(\Gamma y)(x)=y^{\prime}(x)-u(x) y$ is.

Let's define the functions $y_{1}(x)=y(x), y_{2}(x)=(\Gamma y)(x)=y^{\prime}(x)-u(x) y$ and if we write the expression

$$
\ell(y):=-((\Gamma y)(x))^{\prime}+u(x)(\Gamma y)(x)-u^{2}(x) y(x)
$$

on the left side of equation (2.1), we can write the differential equation

$$
\left\{\begin{array}{cc}
y_{1}^{\prime}(x)-u(x) y_{1} & =y_{2} \\
y_{2}^{\prime}(x)+u(x) y_{2}+u^{2}(x) y_{1} & =-k^{2} y_{1}
\end{array}\right.
$$

as a system of differential equations. Similarly (2.2) boundary conditions are

$$
y_{2}(0)-h y_{1}(0)=0, y_{2}^{\prime}(\pi)+H y_{1}(\pi)=0 .
$$

Definition 2.1. (2.3) system of differential equations $y_{1}(\xi)=\alpha_{1}, y_{2}(\xi)=\alpha_{2}, \xi \in[0, \pi], \alpha_{1}, \alpha_{2} \in \mathbb{C}$, providing the initial conditions $y(x)=\left(y_{1}, y_{2}\right)^{t}$ solution $y_{1}(x)$ component (2.1) is called the solution of the equation.

Definition 2.2. The number of $\lambda$ that realizes the existence of the solution of the non-zero $y(x)$ equation of $L y=\lambda y$ is called the eigenvalues of the $L$ problem, and the $y(x)$ function is called the eigenfunction corresponding to these eigenvalues.

In the [1] study, it is shown that the spectrum of the $L$ problem is discrete in case of $u(x) \in B V[0,1]$.

Let us show the solutions of the equation (2.1) with $C(x, \lambda), S(x, \lambda), \varphi(x, \lambda)$ and $\psi(x, \lambda)$, which implement the initial conditions

$$
\begin{aligned}
& C(0, \lambda)=(\Gamma S)(0, \lambda)=1, S(0, \lambda)=(\Gamma C)(0, \lambda)=0 \\
& \varphi(0, \lambda)=1,(\Gamma \varphi)(0, \lambda)=h, \psi(\pi, \lambda)=1,(\Gamma \psi)(\pi, \lambda)=-H .
\end{aligned}
$$

In this case, it is clear that $C(x, \lambda), S(x, \lambda), \varphi(x, \lambda)$ and $\psi(x, \lambda)$ are entaire functions for each fixed $x$ relative to $\lambda$ and

$$
U(\varphi)=0, V(\psi)=0
$$

conditions holds.

Let

$$
\langle y(x), z(x)\rangle:=y(x)(\Gamma z)(x)-(\Gamma y)(x) z(x)
$$

define

$$
\Delta(\lambda)=\langle\psi(x, \lambda), \varphi(x, \lambda)\rangle
$$

function. 
It is clear that the expression $\langle\psi(x, \lambda), \varphi(x, \lambda)\rangle$ does not depend on the variable $x$ by the Liouville formula. The function $\Delta(\lambda)$ is called the characteristic function of the boundary value problem $L=L(u(x), h, H)$.

If we write $x=0$ and $x=\pi$ in the expression (2.5),

$$
\Delta(\lambda)=V(\varphi)=-U(\psi)=(\Gamma \varphi)(\pi, \lambda)+H \varphi(\pi, \lambda)=-(\Gamma \psi)(0, \lambda)+h \psi(0, \lambda)
$$

is obtained. On the other hand, since $\Delta(\lambda)$ is a entire function of $\lambda$, it has a countable number of zero, so the $L$ problem has a countable number of eigenvalues.

Lemma 2.3. The sequence $\left\{\lambda_{n}\right\}_{n \geq 1}$, which is the zeros of the characteristic function $\Delta(\lambda)$, is ordered and is the eigenvalues of the L problem. The functions $\varphi\left(x, \lambda_{n}\right)$ and $\psi\left(x, \lambda_{n}\right)$ are suitable eigenfunctions. There are $\left(\beta_{n}\right)_{n \geq 1}$ sequences so that

equality is achieved.

$$
\psi\left(x, \lambda_{n}\right)=\beta_{n} \varphi\left(x, \lambda_{n}\right), \beta_{n} \neq 0
$$

Proof. Let $\lambda_{o}$ be a zero of the function $\Delta(\lambda)$. In this case, we take the existence of $\beta_{o} \neq 0$ such that $\varphi\left(x, \lambda_{o}\right)=\beta_{o} \psi\left(x, \lambda_{o}\right)$ from the equations (2.4) and (2.6). On the other hand, the functions $\varphi\left(x, \lambda_{o}\right)$ and $\psi\left(x, \lambda_{o}\right)$ provide boundary conditions (2.2). Here, it is obtained that $\lambda_{o}$ has eigenvalue, eigenfunctions corresponding to $\varphi\left(x, \lambda_{o}\right)$ and $\psi\left(x, \lambda_{o}\right)$ functions.

Conversely, let $\lambda_{o}$ be the eigenvalues of the $L$ problem, and $y_{o}(x)$ is the corresponding eigenfunction. In this case, $U\left(y_{o}\right)=V\left(y_{o}\right)=0$ is. Also, since $y_{o}(x) \neq 0$ is $y_{o}(0)=1,\left(\Gamma y_{o}\right)(0)=h$, so $y_{o}(x) \equiv \varphi\left(x, \lambda_{o}\right)$ is obtained. Here, (2.6) is used, $\Delta\left(\lambda_{o}\right)=V\left(\varphi\left(x, \lambda_{o}\right)\right)=V\left(y_{o}(x)\right)=0$ is obtained.

Definition 2.4. The sequence of $\left(\alpha_{n}\right)_{n \geq 1}$ defined as

$$
\alpha_{n}:=\int_{0}^{\pi} \varphi^{2}\left(x, \lambda_{n}\right) d x
$$

is called normalized numbers of $L$ problem, and $\left\{\lambda_{n}, \alpha_{n}\right\}$ is called spectral data.

\section{Lemma 2.5 .}

are correct. Here, $\dot{\Delta}(\lambda)=\frac{d}{d \lambda} \Delta(\lambda)$ is.

$$
\beta_{n} \alpha_{n}=-\dot{\Delta}(\lambda)\left(\lambda_{n}\right), \quad(n=1,2, \ldots)
$$

Proof. Since

(2.6) using the equation,

$$
\frac{d}{d \lambda}\left\langle\psi(x, \lambda), \varphi\left(x, \lambda_{n}\right)\right\rangle=\left(\lambda-\lambda_{n}\right) \psi(x, \lambda) \varphi\left(x, \lambda_{n}\right)
$$

$$
\begin{aligned}
\left(\lambda-\lambda_{n}\right) \int_{0}^{\pi} \psi(x, \lambda) \varphi\left(x, \lambda_{n}\right) d x & =(\Gamma \varphi)\left(\pi, \lambda_{n}\right)+H \varphi(\pi, \lambda)+(\Gamma \psi)(0, \lambda)-h \psi(0, \lambda) \\
& =\Delta\left(\lambda_{n}\right)-\Delta(\lambda)
\end{aligned}
$$

is obtained. From here, while $\lambda \rightarrow \lambda_{n}$

$$
\int_{0}^{\pi} \psi\left(x, \lambda_{n}\right) \varphi\left(x, \lambda_{n}\right) d x=\dot{\Delta}\left(\lambda_{n}\right)
$$

is. If we use (2.7) and (2.8), we get (2.9).

Lemma 2.6. The eigenvalues $\lambda_{n}(n=1,2, \ldots)$ of the L problem are real and simple.

The proof of the Lemma is similar to the proof of Lemma 4 in [2].

Theorem 2.7. While $|\rho| \rightarrow \infty$, for the solution of $\varphi(x, \lambda)=\left(\varphi_{1}(x, \lambda), \varphi_{2}(x, \lambda)\right)^{t}$ and $\psi(x, \lambda)=\left(\psi_{1}(x, \lambda), \psi_{2}(x, \lambda)\right)^{t}$ equation (2.1) on $[0, \pi]$ with respect to $x$ uniformly

$$
\varphi_{1}(x, \lambda)=\cos \rho x+O\left(\frac{1}{|\rho|} \exp (|\tau| x)\right)=O(\exp (|\tau| x))
$$




$$
\begin{gathered}
\left(\Gamma \varphi_{1}\right)(x, \lambda)=\varphi_{2}(x, \lambda)=-\rho \sin \rho x+O(\exp (|\tau| x))=O(\exp (|\tau| x)) \\
\psi_{1}(x, \lambda)=\cos \rho(\pi-x)+O(\exp (|\tau|(\pi-x)))=O(\exp (|\tau|(\pi-x))) \\
\psi_{2}(x, \lambda)=\left(\Gamma \psi_{1}\right)(x, \lambda)=\rho \sin \rho(\pi-x)+O(\exp (|\tau|(\pi-x)))=O(|\rho| \exp (|\tau|(\pi-x)))
\end{gathered}
$$

behavior is valid. Here is $\lambda=\rho^{2}, \tau=\operatorname{Im} \lambda$.

Proof. Since the function $u(x)$ has a boundadly variation on $[0, \pi]$, it is summable in this interval.

Therefore, if we use the appropriate theorem in the [6] study, (2.3) the system of differential equations has a one solution $\left(\varphi_{1}(x, \lambda), \varphi_{2}(x, \lambda)\right)^{t}$ which realizes the initial conditions $\varphi_{1}(0, \lambda)=1, \varphi_{2}(0, \lambda)=h$. In this case, the system of

$$
\begin{aligned}
\varphi_{1}(x, \lambda) & =\cos \rho x+\frac{h}{\rho} \sin \rho x \\
& +\int_{0}^{x}\left\{u(t) \varphi_{1}(t, \lambda) \cos \rho(x-t)-\left[u^{2}(t) \varphi_{1}(t, \lambda)+u(t) \varphi_{2}(t, \lambda)\right] \frac{\sin \rho(x-t)}{\rho}\right\} d t \\
\varphi_{2}(x, \lambda) & =-\rho \sin \rho x+h \cos \rho x \\
& +\int_{0}^{x}\left\{-\rho u(t) \varphi_{1}(t, \lambda) \sin \rho(x-t)-\left[u^{2}(t) \varphi_{1}(t, \lambda)+u(t) \varphi_{2}(t, \lambda)\right] \cos \rho(x-t)\right\} d t
\end{aligned}
$$

integral equations has only one solution.

Let's define

$$
\varphi_{1}^{*}(x, \lambda)=\left|\varphi_{1}(x, \lambda)\right| \exp (-|\tau| x), \varphi_{2}^{*}(x, \lambda)=\left|\varphi_{2}(x, \lambda)\right| \exp (-|\tau| x)
$$

functions. Considering that

$$
|\sin \rho x| \leq \exp (|\tau| x),|\cos \rho x| \leq \exp (|\tau| x), u(x), u^{2}(x) \in L_{1}[0, \pi],
$$

and Gronwal's theorem given in the study [1],

$$
\begin{gathered}
\varphi_{1}^{*}(x, \lambda) \leq K\left(1+\frac{|h|}{|\rho|}\right) \exp \left(\frac{1}{|\rho|} \int_{0}^{x}|u(t)|^{2} d t\right) \\
\frac{1}{|\rho|} \varphi_{2}^{*}(x, \lambda) \leq K\left(1+\frac{|h|}{|\rho|}\right) \exp \left(\frac{1}{|\rho|} \int_{0}^{x}|u(t)|^{2} d t\right)
\end{gathered}
$$

inequality for each $x \in[0, \pi]$ is obtained. Here is $K=\exp \left(2 \int_{0}^{x}|u(t)| d t\right)$. From here, the behavior of $|\rho|$ for sufficiently large values (2.10) and (2.11) is obtained. Similarly (2.12) and (2.13) behavior is shown to be valid.

Remark 2.8. Similarly, while $|\rho| \rightarrow \infty$,

$$
\begin{gathered}
\varphi_{1}(x, \lambda)=\cos \rho x+u(x) \frac{\sin \rho x}{2 \rho}-\frac{1}{2 \rho} \int_{0}^{x} \sin \rho(x-2 t) d u(t)+O\left(\frac{1}{|\rho|} \exp (|\tau| x)\right) \\
\left(\Gamma \varphi_{1}\right)(x, \lambda)=\varphi_{2}(x, \lambda)=-\rho \sin \rho x-u(x) \cos \rho x-\frac{1}{2} \int_{0}^{x} \cos \rho(x-2 t) d u(t)+O(\exp (|\tau| x))
\end{gathered}
$$

asymptotic behaviors are valid for each $x \in[0, \pi]$.

Theorem 2.9. The $\left\{\lambda_{n}\right\}_{n \geq 0}$ set of eigenvalues of the problem $L$ can be counted and

$$
\begin{gathered}
\rho_{n}=\sqrt{\lambda_{n}}=n+\frac{\omega}{\pi n}+\frac{K_{n}}{n}, \quad\left\{K_{n}\right\} \in \ell_{2} . \\
\varphi_{1}\left(x, \lambda_{n}\right)=\cos n x+\frac{\xi_{1, n}(x)}{n} \int_{0}^{\pi} u(t) \sin 2 n t d t+\frac{\xi_{2, n}(x)}{n},\left|\xi_{1, n}(x)\right|,\left|\xi_{2, n}(x)\right| \leq c
\end{gathered}
$$


asymptotic behavior is valid when $n \rightarrow \infty$. Here is

$$
\omega=H+h-\frac{1}{2} u(\pi)-\frac{1}{2} \int_{0}^{\pi} u^{2}(t) d t .
$$

Proof. $\varphi_{1}(x, \lambda)$ and $\varphi_{2}(x, \lambda)$ functions (2.10) and (2.11) by taking advantage of the behavior given (2.14) can write the equation as

$$
\begin{gathered}
\varphi_{1}(x, \lambda)=\cos \rho x+\int_{0}^{x} u(t) \cos \rho(x-2 t) d t-h \int_{0}^{x} u(t) \frac{\sin \rho(x-2 t)}{\rho} d t \\
-\int_{0}^{x} u^{2}(t) \frac{\sin \rho(x-2 t)}{2 \rho} d t+\left(h-\frac{1}{2} \int_{0}^{x} u^{2}(t) d t\right) \frac{\sin \rho x}{\rho}+O\left(\frac{e^{|\tau| x}}{|\rho|}\right) \\
\left(\Gamma \varphi_{1}\right)(x, \lambda)=\varphi_{2}(x, \lambda)=-\rho \sin \rho x-\rho \int_{0}^{x} u(t) \sin \rho(x-2 t) d t \\
-h \int_{0}^{x} u(t) \cos \rho(x-2 t) d t-\frac{1}{2} \int_{0}^{x} u^{2}(t) \cos \rho(x-2 t) d t \\
+\left(h-\frac{1}{2} \int_{0}^{x} u^{2}(t) d t\right) \cos \rho x+O\left(e^{|\tau| x}\right) .
\end{gathered}
$$

Since $u(x), u^{2}(x) \in B V[0, \pi]$,

$$
\Delta(\lambda)=-\rho \sin \rho \pi+\omega \cos \rho \pi+K(\rho)
$$

for the $\Delta(\lambda)=\left(\Gamma \varphi_{1}\right)(\pi, \lambda)+H \varphi_{1}(\pi, \lambda)$ characteristic function is used if

$$
\begin{aligned}
& \left|\int_{0}^{x} u(t) \sin \rho(x-2 t) d t\right|=O\left(\frac{1}{|\rho|}\right), \quad\left|\int_{0}^{x} u(t) \cos \rho(x-2 t) d t\right|=O\left(\frac{1}{|\rho|}\right) \\
& \left|\int_{0}^{x} u^{2}(t) \sin \rho(x-2 t) d t\right|=O\left(\frac{1}{|\rho|}\right), \quad\left|\int_{0}^{x} u^{2}(t) \cos \rho(x-2 t) d t\right|=O\left(\frac{1}{|\rho|}\right)
\end{aligned}
$$

evaluations are used due to the property of the Stiltijes integral is obtained. Here, let's get

$$
\begin{gathered}
\omega=H+h-\frac{1}{2} u(\pi)-\frac{1}{2} \int_{0}^{\pi} u^{2}(t) d t, \\
K(\rho)=-\frac{1}{2} \int_{0}^{\pi} \cos \rho(x-2 t) d u(t)+O\left(\frac{1}{|\rho|}\right) \\
G_{\delta}=\{\rho:|\rho-K| \geq \delta, \delta>0, \quad K=0, \pm 1, \pm 2, \ldots\} .
\end{gathered}
$$

In this case, if we use Lemma3.2 in [8], we obtain the existence of the number $\rho^{*}>0$ such that

$$
|\Delta(\lambda)| \geq c_{\delta}|\rho| \exp (|\tau| \pi), \rho \in G_{\delta}, \quad|\rho| \geq \rho^{*}
$$

is.

Let $\Gamma_{n}=\left\{\lambda:|\lambda|=\left(n+\frac{1}{2}\right)^{2}\right\}$. So if we apply the Rouche theorem, take that $\Gamma_{n}$ has exactly $(n+1)$ zeroes in the function $\Delta(\lambda)$ and each circle $\gamma_{n}(\delta)=\{\rho:|\rho-n| \leq \delta\}$ has a single zero in the sufficiently large values of $n$. Since $\delta>0$ is arbitrary, we take the equation

$$
\rho_{n}=n+\varepsilon_{n}, \varepsilon_{n}=o(1), n \rightarrow \infty
$$


If (2.17) is substituted in the (2.16) function,

$$
0=\Delta\left(\rho_{n}^{2}\right)=-\left(n+\varepsilon_{n}\right) \sin \left(n+\varepsilon_{n}\right) \pi+\omega \cos \left(n+\varepsilon_{n}\right) \pi+K_{n}
$$

is. From here,

$$
\varepsilon_{n}=\left(H+h-\frac{1}{2} u(\pi)-\frac{1}{2} \int_{0}^{\pi} u^{2}(t) d t\right) \frac{1}{\pi n}-\frac{1}{2 \pi n} \int_{0}^{\pi} \cos n(\pi-2 t) d u(t)+O\left(\frac{1}{n^{2}}\right)
$$

is obtained. Therefore, $\rho_{n}=n+\frac{\omega}{\pi n}+\frac{K_{n}}{n}$ asymptotic expression is obtained. Here,

$$
K_{n}=-\frac{1}{2} \int_{0}^{\pi} \cos n(\pi-2 t) d u(t)+O\left(\frac{1}{n}\right)
$$

and

are.

$$
\sum_{n} K_{n} \cos n x=K(x) \in B V[0, \pi]
$$

If the expression of $\rho_{n}$ is written in its place (2.15),

$$
\varphi_{1}\left(x, \rho_{n}\right)=\cos n x+\frac{\sin n x}{n}\left[h+h_{o}(x)\right]-\varepsilon_{n} x \sin n x+\frac{1}{n} \int_{0}^{x} u(t) \sin n(x-2 t) d t+O\left(\frac{1}{n^{2}}\right)
$$

asymptotic behavior is obtained for the eigenfunctions of the given problem. Here is

$$
h_{o}(x)=h \int_{0}^{x} u(t) \cos n(x-2 t) d t-\frac{1}{2} \int_{0}^{x} u^{2}(t) \cos n(x-2 t) d t-\frac{1}{2} \int_{0}^{x} u^{2}(t) d t .
$$

(2.18) by squaring the equation is integrated on $[0, \pi]$ of the given problem $\left(\alpha_{n}\right)_{n \geq 0}$ for the normalized numbers,

$$
\alpha_{n}=\int_{0}^{\pi} \varphi_{1}^{2}\left(x, \rho_{n}\right) d x=\frac{\pi}{2}+a_{n}
$$

asymptotic expression is obtained. Here the sequence $\left(a_{n}\right)_{n \geq 0}$ satisfies the condition

$$
\sum_{n} a_{n} \cos n x=a(x) \in B V[0, \pi]
$$

\section{CONFLICTS OF INTEREST}

The authors declare that there are no conflicts of interest regarding the publication of this article.

\section{REFERENCES}

[1] Amirov, R.Kh., Guseinov, I.M., Boundary Value Problems for a class of Sturm-Liouville operators with Nonintegrable Potential, Diff. Equations, 38(8)(2002), 1195-1197. 1, 2, 2

[2] Amirov, R.Kh., Çakmak, Y., Gülyaz, S., Boundary value problem for second-order differential equations with coulomb singularity on a finite interval, Indian J. Pure Appl. Math., 37(3)(2006), 125-140. 1, 2

[3] Amirov, R., Ergun, A., Durak, S., Half inverse problems for the quadratic pencil of the Sturm-Liouville equations with impulse, Numerical Methods for Partial Differential Equations, DOI: 10.1002/num.22559, (2020). 1

[4] Amirov, R.Kh., Ergun, A., Direct and inverse problems for diffusion operatör with discontinuity points, TWMS J. App. Eng. Math. 9(1)(2019), 9-21. 1

[5] Ergun, A., Integral representation for solution of discontinuous diffusion operator with jump conditions, Cumhuriyet Science Journal, 39(4)(2018), 842-863. 1

[6] Naimark, M.A., Lineer differential operators, Moscow, Nauka, 1969. 2

[7] Savchuk, A.M., Shkalikov, A.A., Sturm-Liouville operators with singular potentials, Math. Zametki, 66(1999), 897-912. 1

[8] Shkalikov, A.A., Boundary value problems for the ordinary differential equations with the parameter in the boundary conditions, Trudy Sem. I. G. Petrovskogo, 9(1983), 190-229. 2.9 